\title{
Testing for uniformity of UHECR arrival directions
}

\author{
Anatoly Ivanov* \\ Shafer Institute for Cosmophysical Research \& Aeronomy \\ 31 Lenin Ave., Yakutsk, 677980, Russian Federation \\ E-mail: ivanoveikfia.ysn.ru
}

\begin{abstract}
Arrival directions of ultra-high energy cosmic rays (UHECRs) exhibit mainly isotropic distribution with a hint of small deviations in particular energy bins. In this paper the Yakutsk array data are tested for circular uniformity of arrival directions using two methods appropriate for the energy ranges below and above $10^{18} \mathrm{eV}$. No statistically significant deviation from uniformity is found in arrival directions of cosmic rays (CRs) detected within the observational period 19742000 .
\end{abstract}

The 34th International Cosmic Ray Conference,

30 July- 6 August, 2015

The Hague, The Netherlands

\footnotetext{
${ }^{*}$ Speaker.
} 


\section{Introduction}

The widely used technique to search for large scale anisotropies in arrival directions of CRs is the analysis in right ascension (RA), using harmonic analysis or another convenient method. The greatest difficulty in this approach consists in the evaluation of the directional exposure of the experiment which is distorted by the instrumental errors and weather conditions. In the case of the Yakutsk Array data, only at energies above $1 \mathrm{EeV}=10^{18} \mathrm{eV}$ these effects appear marginal as compared with statistical errors in estimation of extensive air shower (EAS) parameters rising with energy.

Accordingly, we divide our target energy range into two parts, below and above $E_{t h r}=1 \mathrm{EeV}$, where different methods of analysis are applicable. In practice, the measurement below $E_{t h r}$ is complicated by the need of correcting the counting rate for instrumental and atmospheric effects, that must be done to prevent the introduction of artificial variations in the CR flux.

For example, the East-West method, being based on a differential technique, was designed to avoid introducing such corrections, preventing the possible associated systematics to affect the results. The original idea was proposed in [四] and applied to the data of the Mt. Norikura array.

In this paper we use another approach, named the South-North method (SNM), which is based on the comparison of the data samples in independent right ascension circles. The principle of the method and results of SNM application to the Yakutsk Array data are given in Section 2.

Above $E_{t h r}$ the array exposure can be assumed uniform in RA, and we use here a version of harmonic analysis adapted to test for uniformity of the distribution of the first harmonic phase. The method is described in Section 3.

A subset of the Yakutsk Array data consisting of 590887 EAS events in the energy range $E>0.1 \mathrm{EeV}$ with zenith angles $\theta<60^{\circ}$ detected within the array area during the observational period $1974-2000$ is used in analysis. The rest of the data will be analyzed later.

\section{2. $E<10^{18} \mathbf{e V}$. The South-North method}

Earth rotation gives the possibility to scan celestial regions providing the right ascension distribution of CR arrival directions. Scintillation detectors of the Yakutsk Array have 24 hour duty cycle resulting in almost uniform directional exposure. However, at least at energies below $10^{18}$ $\mathrm{eV}$, there are deviations from the uniformity which are caused by the detector maintenance and variations of the atmospheric conditions. Estimation of the diurnal and seasonal variations of the Yakutsk Array exposure was given in [[]].

In this paper, however, we do not use estimations of the array exposure and corrections. Instead, the RA distribution of CRs within given declination interval is tested for uniformity as is. The main assumption we are basing is that there is only one discerned source of CRs vs isotropic background in the data observed, if any; and it is restricted in angular size. Dividing a bulk of the data into samples within declination bins, say, $\delta \leq 0$ and $\delta>0$, one can compare RA distributions using $\chi^{2}$-test. An advantage of the method is the same instrumental and weather non-uniformities of the distributions observed in different declination bins.

If there is no significant deviation of the samples from each other, one can conclude that: i) distributions are uniform (taking into account the array exposure); ii) or the same source is present 
in both samples. Restricted angular size of the source excludes the second alternative, particularly, in the case of three or more samples.

\subsection{Statistical power of SNM}

The statistical power of the method is its efficiency depending on the sample size $N$. We have to find a lower limit of $N$ needed to reject the isotropic hypothesis, $H_{0}$, at a confidence level of $99 \%$ when an alternative hypothesis, $H_{1}$, is true. To estimate $N_{\min }$, we used $H_{1}$ consisting of a separate CR source as a $\delta$-function located in $\alpha_{0}$, yielding the fraction $f$ of the total CR flux, and isotropic background which provides $(1-f)$ of the flux. The second sample consists of the isotropic CRs.

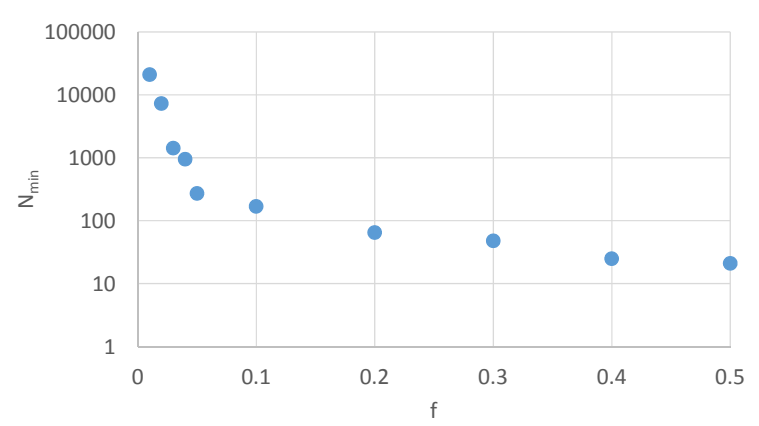

Figure 1: Statistical power of the South-North method. The minimal sample size, $N_{\min }$, needed to reject a null hypothesis if there is a separable source of CRs giving a fraction, $f$, of the total flux.

Monte Carlo algorithm is used to model $H_{0}$ and $H_{1}$ with the Yakutsk Array exposure. The result of SNM simulation is illustrated in Fig. $\mathbb{}$.

\subsection{Application of SNM to the Yakutsk Array data}

Right ascension distributions of CR arrival directions are sampled in energy, $\Delta \lg (E)=0.25$, and declination bins, $\Delta \delta=30^{\circ}$. The energy limits suitable for the South-North method are $10^{17}<$ $E<10^{18} \mathrm{eV}$. The resultant number of samples are 4 in energy, and 3 in declination. RA interval is divided into $N_{R A}=9$ equidistant bins.

In a given energy interval, three samples of RA distribution (North: $60^{0}<\delta \leq 90^{0}$, Middle : $30^{0}<\delta \leq 60^{0}$, South : $0^{0}<\delta \leq 30^{0}$ ) are compared in pairs using $\chi^{2}$ goodness-of-fit test with 8 degrees of freedom.

\begin{tabular}{|l|r|r|r|}
\hline $\lg (\mathrm{E}, \mathrm{eV})$ bins & Middle-North & Middle-South & South-North \\
\hline $17.00-17.25$ & 5.72 & 73.43 & 78.96 \\
\hline $17.25-17.50$ & 13.22 & 20.87 & 2.20 \\
\hline $17.50-17.75$ & 26.64 & 31.04 & 6.03 \\
\hline $17.75-18.00$ & 3.85 & 1.18 & 49.49 \\
\hline
\end{tabular}

Table 1: Chance probability (in \%) of the two samples of RA distribution to be consistent.

In Table 1 the resultant probabilities, $P$, are given for the $\chi^{2}$ random variable to be equal or greater than the test-statistic of the pair of samples. In the case $P<1 \%$ two samples are significantly different. It is not the case for all samples in energy and declination bins. 
One can conclude that three samples: North,Middle, South in declination bins are compatible, so there is no separable source of CRs with the angular size below $30^{0}$ in four energy intervals considered.

\section{3. $E>10^{18} \mathrm{eV}$. Double harmonic analysis}

Harmonic analysis in RA of CR arrival directions detected with the Yakutsk Array before 2000 had found no statistically significant deviations from isotropic distribution [B]]. However, the first harmonic phase exhibits a non-uniform behavior: gradual increase with energy in RA, at least in the energy interval above $10 \mathrm{EeV}$ [䧃]. While the most convenient way of testing for uniformity of arrival directions is analysis of the minimum width of the distribution [可], we are using here another approach aimed at the phase behavior, namely, harmonic analysis of the phases - in other words, double harmonic analysis of the RA distribution.

The phase of the first harmonic points to the excess flux of CRs in RA distribution, if there is a source, or is uniformly distributed otherwise. It's natural to apply the Rayleigh test to find the possible non-uniformity of the phase. In this way, we have to divide the data in the given energy interval into a set of $M$ sub-samples with $n$ EAS events. A set of $M$ directions formed by the phases of the first harmonics in sub-samples, in turn, gives the first harmonic amplitude, $A_{1}$. The Rayleigh probability $P\left(>A_{1}\right)=\exp \left(-\frac{M A_{1}^{2}}{4}\right)$ states the isotropic amplitude to be larger than the observed $A_{1}$ by chance.

The Yakutsk Array data consisting of CRs detected before 2000 in four energy intervals divided into sub-samples of size $M=[\sqrt{N}]$, where $N$ is the number of CRs in the energy interval. The phases, $\phi_{1}$, of the first harmonic amplitudes of $n=[N / M]$ points in RA distribution are the input data for harmonic analysis of phases. The results are given in Table 2 and Fig. $\square$.

\begin{tabular}{|l|r|r|r|r|r|r|}
\hline $\lg (\mathrm{E}, \mathrm{eV})$ bins & $N$ & $M$ & $\left\langle\phi_{1}^{0}\right\rangle$ & $\delta \phi_{1}^{0}$ & $A_{1}, \%$ & $P, \%$ \\
\hline $18.0-18.5$ & 28136 & 168 & 2.9 & 102.4 & 2.2 & 98.1 \\
\hline $18.5-19.0$ & 4141 & 64 & -5.2 & 102.2 & 16.3 & 65.4 \\
\hline $19.0-19.5$ & 437 & 21 & 33.2 & 76.4 & 73.3 & 5.9 \\
\hline $19.5-20.0$ & 55 & 7 & 47.2 & 91.6 & 64.4 & 48.4 \\
\hline
\end{tabular}

Table 2: Double harmonic analysis of RA distribution detected before 2000 in Yakutsk.

Rayleigh test results in uniform distribution of phases in all energy intervals $(P>1 \%)$. However, r.m.s. deviations of phases, $\delta \phi_{1}$, at energies above $10^{19} \mathrm{eV}$ are somewhat below the value $103.9^{0}$ expected for isotropy. It was analyzed in detail previously [䧃]. The mean value of the phase is $33.2^{0} \pm 76.4^{0}$ in the energy interval $10<E<31.6 \mathrm{EeV}$ in agreement with other measurements [四, 团], while $\phi_{1} \sim 0^{0}$ below $10 \mathrm{EeV}$ is an artefact of the uniform distribution averaged in the interval $\phi_{1} \in\left(-180^{\circ}, 180^{\circ}\right)$.

\section{Conclusions}

The Yakutsk Array data set of CRs detected during observational period 1974 to 2000 is used to analyze arrival directions. Two methods are applied to test for uniformity of the right ascension 


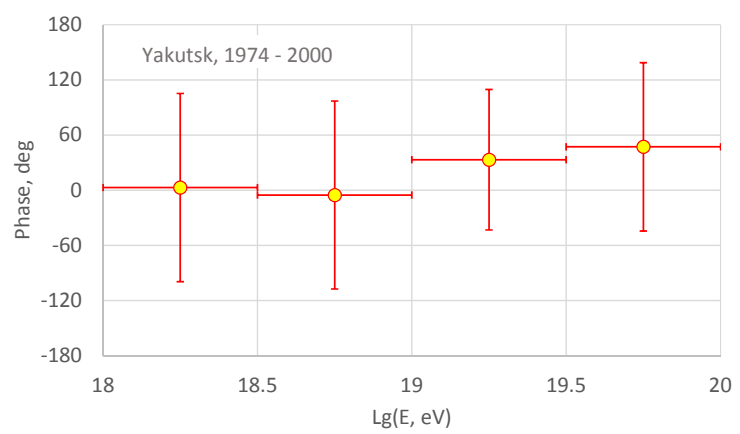

Figure 2: Phase of the first harmonic as a function of energy. Horizontal bars indicate energy bins, vertical bars are r.m.s. deviations of phases within bins.

distribution: the South-North method at energies below $E_{t h r}=10^{18} \mathrm{eV}$ where the diurnal and seasonal variations of the array exposure are unavoidable, and double harmonic analysis above the threshold.

At $E<E_{t h r}$ three samples of RA distribution in different declination bins are compatible. The conclusion is that there is no single separable source of CRs with the angular size below $30^{\circ}$ detectable in four energy intervals considered.

Above $E_{t h r}$ the first harmonic phase exhibits the RA distribution having no statistically significant deviation from isotropy in energy intervals of the width $\Delta \lg E=0.5$.

\section{Acknowledgments}

I'm grateful to the Yakutsk Array staff for the data acquisition and analysis. The work is supported by RFBR grant no. 13-02-12036.

\section{References}

[1] K. Nagashima et al., Il Nuovo Cimento C 12 (1989) 695

[2] M.I. Pravdin et al., JETP 92 (2001) 766

[3] V.P. Egorova et al., J. Phys. Soc. Japan B 70 (2001) 9

[4] A.A. Ivanov et al., $A p P h 62$ (2015) 1

[5] A.A. Ivanov, ApJ 804 (2015) 122

[6] I. Sidelnik et al., Proc. of $33^{d}$ ICRC, Rio de Janeiro (2013) icrc2013-0739 